\title{
The safety of available treatments options for neuroendocrine tumors
}

\section{A. Faggiano, F. Lo Calzo, G. Pizza, R. Modica \& A. Colao}

To cite this article: A. Faggiano, F. Lo Calzo, G. Pizza, R. Modica \& A. Colao (2017): The safety of available treatments options for neuroendocrine tumors, Expert Opinion on Drug Safety, DOI: 10.1080/14740338.2017.1354984

To link to this article: http://dx.doi.org/10.1080/14740338.2017.1354984

Accepted author version posted online: 14 Jul 2017. Published online: 20 Jul 2017.

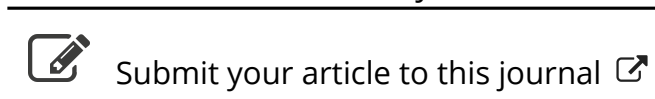

Џ Article views: 46

Q View related articles ¿ View Crossmark data ¿ 


\title{
The safety of available treatments options for neuroendocrine tumors
}

\author{
A. Faggiano ${ }^{\text {a }}$, F. Lo Calzo ${ }^{\text {b }}$ G. Pizza ${ }^{\text {, }}$ R. Modica ${ }^{\text {b }}$ and A. Colao ${ }^{\text {b }}$ \\ aThyroid and Parathyroid Surgery Unit, Istituto Nazionale per lo studio e la cura dei tumori "Fondazione G. Pascale" - IRCCS, Naples, Italy; \\ bDepartment of Clinical Medicine and Surgery, Federico II University, Naples, Italy
}

\section{ABSTRACT}

Introduction: Neuroendocrine neoplasms (NEN) represent a heterogeneous group of malignancies generally characterized by low proliferation and indolent course. However, about half of the newly diagnosed cases are metastatic and require long-term systemic therapies.

Areas covered: This review revises the literature to summarize the current knowledge upon safety of all systemic treatment options available. Thirty three different clinical studies have been considered, including 4 on somatostatin analogues (SSA), 5 on targeted therapies, 10 on peptide receptor radionuclide therapy (PRRT), and 14 on chemotherapy.

Expert opinion: SSA are safe and well tolerated without any relevant severe adverse event and very low treatment discontinuation rate. Targeted therapies show a satisfying safety profile. Most adverse events are grade 1-2 and easy manageable with dose reduction or temporary interruption. PRRT is manageable and safe with a low rate of grade 3-4 adverse events. However, severe renal and hematologic toxicity may occur. Chemotherapy is usually considered after previous therapeutic lines. Therefore, these subjects are more susceptible to experience adverse events due to cumulative toxicities or poor performance status.

The available systemic treatment options are generally well tolerated and suitable for long-term administration. Cumulative toxicity should be taken in account for the definition of therapeutic sequence.
ARTICLE HISTORY

Received 25 April 2017

Accepted 10 July 2017

KEYWORDS

Neuroendocrine neoplasm; safety; somatostatin analogues; chemotherapy; targeted therapy; PRRT

\section{Introduction}

Neuroendocrine neoplasms (NEN) represent a heterogeneous group of malignancies, arising from the neuroendocrine system and showing variable histology and clinical behavior. The gastrointestinal $(67 \%)$ or the bronchopulmonary tract $(25 \%)$ represent the predominant sites of localization of NEN. In the last years the incidence has increased to 7.4 cases $/ 100,000$. However, about $50 \%$ of newly diagnosed patients present with metastasis, requiring an effective systemic treatment to prolong survival. The 5year survival rate for this population is about $40 \%$ [1]. The most recent classification of NEN is the WHO (World Health Organization) classification published in 2010 [2]. According to WHO classification, gastroenteropancreatic neuroendocrine neoplasms (GEP NEN) can be divided into well differentiated neoplasms graded, according to Ki67 index, G1 (Ki67 $\leq 2 \%$ ) or G2 (Ki67 3-20\%), and poorly differentiated neoplasms graded G3 (Ki67 > 20\%). The landscape of the therapeutic options in NEN has considerably expanded in the last years, thanks to the growing knowledge of tumor cell biology and to the increased interest in the molecular changes involved in development and progression of cancer. The current systemic therapies for locally advanced or metastatic G1/G2 NEN include somatostatin analogues (SSA), interferon- $\mathrm{a}(\mathrm{IFN}-\mathrm{a})$, radionuclide ${ }^{90} \mathrm{Yttrium}\left({ }^{90} \mathrm{Y}\right)$ or ${ }^{177}$ Lutetium $\left({ }^{177} \mathrm{Lu}\right)$ coupled with SSA, targeted therapies, chemotherapy. Cytotoxic chemotherapy is also the main therapeutic approach for patients with highly proliferating NEN $(G 3)[3,4]$.
Treatment decision in NEN should take into account tumor differentiation and related symptoms (mass effect, hormone production) and patients' characteristics, as age, performance status and life expectancy. In the choice of the therapy, care must be taken to the quality of life of patients, while aiming at controlling tumor growth and secretion, balancing potential risks and benefits. Thus, drug safety represents a hot topic in this field. In this light careful attention must be taken, because of potential drugrelated toxicity in patients who often are expected be treated for long periods, since most NEN patients have good survival rate. Furthermore, possible toxicity due to different drug interactions and the route of administration must be considered. In line with the literature data, and with the main clinical international trials, we conducted this review to appraise critically the safety profile of the different therapeutic strategies in the management of NEN. For SSA and targeted therapy, prospective phase II and III studies are reported. For peptide receptor radionuclide therapy (PRRT) and chemotherapy data are derived from clinical trials in NEN if available, or from reports of retrospective NEN series.

\section{Somatostatin analogues (SSA)}

Octreotide and lanreotide are effective in controlling most NEN endocrine syndromes. Their antiproliferative effect has been recognized since the publication of the PROMID study in 2009, the first randomized trial with SSA in NEN, evaluating the effects of octreotide long-acting release (LAR) in 


\section{Article highlights}

- Biotherapy with somatostatin analogues, targeted therapies with mTOR and tyrosine-kinase inhibitors, peptide receptor radionuclide therapy and chemotherapy represent well established systemic therapies for neuroendocrine neoplasms.

- The available systemic treatment options for neuroendocrine neoplasms are generally well tolerated and suitable for long-term administration.

- Somatostatin analogues are safe, manageable and generally well tolerated. Treatment discontinuation as well as dose adjustment are rarely required.

- Adverse events related to targeted therapies can be for the most managed and prevented, sometimes decreasing the dose or temporarily interrupting the administration and rarely discontinuing the drug.

- ${ }^{177}$ Lutetium peptide receptor radionuclide therapy, recently investigated in a phase III clinical trial, is associated with a low rate of serious adverse events. Renal and hematologic function should be long-time monitored because of the possible occurrence of renal insufficiency and myelodysplastic syndrome.

- Cumulative toxicities and poor performance status could explain higher rate of chemotherapy-related serious adverse events.

- Cumulative toxicity should be taken in account for the definition of therapeutic sequence.

This box summarizes key points contained in the article.

metastatic midgut NEN [5]. Nowadays, current NEN guidelines report that SSA are the first therapeutic option in patients with G1-G2 NEN [3]. In clinical practice, SSA are extensively employed, not only for their proved antitumor efficacy but also for their excellent manageability and patients' tolerance. The treatment with long-acting preparations of SSA consists in an intramuscular injection every 2 or 4 weeks (octreotide LAR 10-30 mg; lanreotide autogel 60-120 mg). These agents exhibit a good safety profile, although some adverse events might occur. The most common include abdominal pain with cramps, constipation, diarrhea, steatorrhea, injection site irritation and local pain, nausea and vomiting. Less frequent adverse events are hypothyroidism, cholecystitis and cholelithiasis. Acute pancreatitis, alopecia, acute hepatitis, hyperbilirubinemia, hyperglycemia, hypoglycemia, prolonged QT interval and arrhythmias are rare but still possible complications [6]. A relevant adverse effect is the development of gallstones, in up to $60 \%$ of patients, deriving from inhibition of cholecystokinin release which in postprandial induces emptying of the gallbladder [7]. The prolongation of the QT interval on ECG was observed in patients with acromegaly treated with octreotide. Lanreotide-induced bradycardia is currently limited to one case report [8]. Colao et al. showed in acromegalic patients treated with SSA in first line for 12 months, a worsening of glucose tolerance at the beginning of the treatment in patients with diabetes or glucose intolerance at baseline [9]. In PROMID study, treatment related deaths did not occur. Serious adverse events were observed in 11 patients treated and 10 in placebo group, including gastrointestinal symptoms, hematopoietic alterations and general health status (fatigue or fever); in five treated patients the discontinuation of the treatment occurred. The most frequently observed adverse events with octreotide included diarrhea and flatulence [5]. A similar safety profile was observed for lanreotide. In CLARINET study adverse events were reported in $88 \%$ of treated patients and $90 \%$ in placebo group. Only $50 \%$ of treated patients showed adverse events related to treatment and in $3 \%$ of patients treatment was discontinued. The most common adverse event was diarrhea, followed by abdominal pain, cholelithiasis, flatulence, injection site pain, nausea, vomiting, headache, lethargy, hyperglycemia, decreased levels of pancreatic enzyme [10]. The difference between PROMID and CLARINET studies in the safety profile are reported in Table 1. There is a general trend supporting the use of high doses of octreotide LAR or lanreotide autogel to control symptoms and tumor progression in patients with NEN, after progression under standard SSA dose [11]. According to literature data, the patients treated with high doses SSA showed no difference in common adverse events when compared to standard doses. In particular, Ruszniewski et al., evaluated the effects and safety profile of lanreotide dose titration (60, 90, and $120 \mathrm{mg} / \mathrm{month}$ ), in patients affected by NEN and carcinoid syndrome, after 6 months of treatment. A reduction in $50 \%$ of flushing and diarrhea episodes was observed, and severe adverse events were reported by $10 \%$ of patients; the most common were abdominal pain (38\%), diarrhea (17\%), fatigue (15\%), vomiting (13\%), asthenia (11\%), nausea (10\%), cholelithiasis (10\%) and anorexia (10\%) [12]. Broder et al. reported in a systematic review, that there was not increased toxicity in patients treated with high dose of octreotide LAR when compared with standard doses [13]. Finally, Chadha et al. reported no treatment related toxicities in patients affected by pancreatic NEN (pNEN), treated with octreotide LAR at higher doses (60-90 $\mathrm{mg} /$ month) respect to standard doses (20-30 mg/month) [11]. Reducing the interval between injections, in patients with progressive locally advanced or metastatic NEN, is relatively common in clinical practice. Ferolla et al. showed in 28 patients affected by well differentiated NEN that no additional toxicity was recorded with octreotide LAR $30 \mathrm{mg}$ every 21 days, compared with octreotide LAR $30 \mathrm{mg}$ administered every 28 days; no treatment discontinuation or dose modification were required [14]. Pasireotide is a novel SSA that exhibits a very different binding profile to human somatostatin receptors (SSTR). Although not routinely used in NEN treatment, the safety profile of pasireotide is quite similar to other SSA. Wolin et al. reported in a randomized, multicenter, open-label, phase II study, the

Table 1. Safety profile of SSA from PROMID and CLARINET trials.

\begin{tabular}{lcc}
\hline & PROMID study & $\begin{array}{c}\text { CLARINET study } \\
{[2]}\end{array}$ \\
\hline $\begin{array}{l}\text { Number of patients treated with SSA } \\
\text { Number of treatment related serious }\end{array}$ & $11(26 \%)$ & $3(3 \%)$ \\
$\quad \begin{array}{l}\text { adverse event } \\
\text { Treatment discontinuation because of }\end{array}$ & $5(12 \%)$ & $3(1 \%)$ \\
$\quad$ adverse event & $7(21.9 \%)$ & $26(26 \%)$ \\
Diarrhea & $7(21.9 \%)$ & $14(14 \%)$ \\
Abdominal pain & $4(12.5 \%)$ & $\mathrm{na}$ \\
Flushing & $5(12 \%)$ & $10(10 \%)$ \\
Cholelithiasis & $\mathrm{na}$ & $5(5 \%)$ \\
Hyperglycemia & $5(12 \%)$ & $\mathrm{na}$ \\
Hematopoietic system alterations & $\mathrm{na}$ & $\mathrm{na}$ \\
Treatment related deaths & &
\end{tabular}

SSA: somatostatin analogues, na: not available. 
effects and the safety profile of pasireotide LAR in 48 patients affected by GEP NEN. The patients were treated at different doses of pasireotide LAR: $20 \mathrm{mg}$ (12 patients), $40 \mathrm{mg}$ (14 patients) and $60 \mathrm{mg}$ (16 patients). The most common adverse event reported was diarrhea, followed by fatigue, abdominal pain and nausea; no relationship was observed between the frequency of the adverse events and the dose of pasireotide. Fifteen patients had grade 3-4 adverse events, the most frequent was diabetes mellitus followed by flushing and abdominal pain. Five patients discontinued the treatment early, only one patient developed treatment related severe adverse event (diabetes mellitus complicated by nonketotic hyperosmolar syndrome) [15]. Subsequently a randomized, double blind, phase III study, compared pasireotide LAR with octreotide LAR in managing refractory carcinoid symptoms. Adults with carcinoid tumors of the digestive tract were randomly assigned to receive pasireotide LAR $(60 \mathrm{mg})$ or octreotide LAR (40 mg) every 28 days. In this study, serious adverse events occurred with comparable frequency in the pasireotide LAR (41.5\%) and octreotide LAR (36.8\%) arms, although more serious adverse events in the pasireotide LAR arm were drugrelated $(17 \%$ vs. $3.5 \%)$. The most frequent drug-related adverse events (pasireotide vs. octreotide) included hyperglycemia $(28.3 \%$ vs. $5.3 \%)$, fatigue $(11.3 \%$ vs. $3.5 \%)$, and nausea $(9.4 \%$ vs. $0 \%)[16]$.

\section{Targeted therapy}

Considering the very high rate of patients with synchronous metastases at diagnosis, therefore unsuitable for surgery, and the typical indolent growth of these tumors allowing long survival even in advanced stages of disease, it has become very important to find more effective medical therapy. In this context, the development of targeted therapies for NEN is of extreme interest for their efficacy and for the general good tolerability profile of these drugs, compared, with classical chemotherapy schedules. Mammalian target of rapamycin (mTOR) inhibitor (everolimus) and tyrosine kinase inhibitor (sunitinib), have been approved for the treatment of patients with unresectable or metastatic, progressive well differentiated NEN [17]. Everolimus is an oral mTOR inhibitor that inhibits mTOR kinase and downstream activity, as well as vascular endothelial growth factor (VEGF) and hypoxia inducible factor-1 (HIF-1) expression. mTOR is an intracellular serine/ threonine kinase that forms two functional complexes (mTORC1 and 2) involved in cell survival, proliferation and metabolism, integrating response to glucose, growth factors and hormones. mTOR seems to be involved in the pathogenesis of up to $80 \%$ of human tumors [18], directly controlling cancer cell proliferation and angiogenesis through activation of HIF-1. In particular, mTOR activity is associated with pathogenesis and aggressiveness of pNEN, in which mutations or deregulated expressions of upstream regulators of mTOR have been reported [19]. mTOR inhibitors, originally used to attenuate rejection in solid organ transplantation, have dose limiting toxicities, that include gastrointestinal disturbances, stomatitis, weakness, fatigue, lipid metabolism alterations, leukopenia and thrombocytopenia. In this setting, everolimus showed benefit and safety at daily doses of 1.5 or $3 \mathrm{mg}[20,21]$. In a phase I study, that evaluated everolimus benefit and tolerance in solid tumor therapy, the mean tolerated dose of everolimus was $10 \mathrm{mg} /$ day, and the most common adverse events were rash, fatigue, nausea, vomiting anorexia and stomatitis. Rare adverse events were observed in this context, in particular bronchiolitis obliterans with organizing pneumonia, severe pneumonitis, and diffuse alveolar hemorrhage [22]. Regarding everolimus in NEN, Yao et al., in a phase II study found that grade 1 or 2 adverse events were more common than grade 3 [23]. In the phase II RADIANT-1 trial, all events were grade 1 or 2 , and included stomatitis (45\%), rash $(40 \%)$ and diarrhea (39\%); $8 \%$ of patients developed pneumonitis [24]. In the phase III RADIANT-2 trial, the most common grade 1 or 2 events were stomatitis $(62 \%)$, fatigue $(31 \%)$ and rash (37\%), on the other hand the most common grade 3-4 events included fatigue, diarrhea and hyperglycemia. Furthermore, 5\% of patients presented infections and $12 \%$ pulmonary drug-related adverse events [25]. In the phase III RADIANT-3 trial, the most common adverse events in patients treated with everolimus $10 \mathrm{mg} /$ day, were stomatitis (64\%), rash (49\%), diarrhea (34\%) and infections (23\%). Pneumonitis was observed in $12 \%$ of patients, and atypical infections (such as tuberculosis, reactivation of hepatitis B) was observed in $2 \%$ of patients [26]. In the phase III RADIANT-4 trial, were enrolled patients with well-differentiated non functional lung or gastrointestinal NEN, treated with everolimus $10 \mathrm{mg} / \mathrm{day}$ or placebo. The treatment related adverse events occurred in at least $10 \%$ of patients; the most common were stomatitis (all grades $63 \%$ ), diarrhea and fatigue (all grades $31 \%$ ), infections (all grades 29\%), (Table 2) [27]. Finally, Faggiano et al. reported a good safety profile in patients with extra pancreatic NEN treated with everolimus. In particular, grade 1-2 adverse events were thrombocytopenia and anemia, mucositis and stomatitis, rash, hyperglycemia, diarrhea, and pulmonary events, including pneumonitis, interstitial lung disease, lung infiltrations, and pulmonary fibrosis. No serious adverse events were usually reported and the discontinuation of the treatment was rarely required [28]. Sunitinib is an oral multitarget inhibitor of several tyrosine kinases including VEGF receptors. It has been shown to delay tumor growth in RIP1-Tag2 transgenic mouse model of pancreatic islet-cell tumors [29]. In a phase III trial, oral sunitinib $37.5 \mathrm{mg} /$ day demonstrated a prolonged median progression free survival (PFS) in patients with locally advanced or metastatic well differentiated pNEN [30].

Table 2. Safety profile of everolimus from RADIANT studies.

\begin{tabular}{|c|c|c|c|c|c|c|c|c|}
\hline \multirow[b]{2}{*}{ Grade } & \multicolumn{2}{|c|}{$\begin{array}{c}\text { RADIANT-1 } \\
\text { [1] }\end{array}$} & \multicolumn{2}{|c|}{$\begin{array}{c}\text { RADIANT-2 } \\
{[2]} \\
\end{array}$} & \multicolumn{2}{|c|}{ RADIANT-3 [3] } & \multicolumn{2}{|c|}{$\begin{array}{c}\text { RADIANT-4 } \\
\text { [4] }\end{array}$} \\
\hline & $\begin{array}{l}1-2 \\
(\%)\end{array}$ & $\begin{array}{r}3-4 \\
(\%)\end{array}$ & $\begin{array}{l}1-2 \\
(\%)\end{array}$ & $\begin{array}{l}3-4 \\
(\%)\end{array}$ & $\begin{array}{l}1-2 \\
(\%)\end{array}$ & $\begin{array}{l}3-4 \\
(\%)\end{array}$ & $\begin{array}{l}1-2 \\
(\%)\end{array}$ & $\begin{array}{l}3-4 \\
(\%)\end{array}$ \\
\hline Stomatitis & 45 & na & 62 & na & 64 & na & 54 & 9 \\
\hline Diarrhea & 39 & na & na & 5 & 34 & na & 23.7 & 15 \\
\hline Fatigue & na & na & 31 & na & na & na & 27.2 & 12.3 \\
\hline Rash & 40 & na & 37 & na & 49 & na & 26.7 & 6.4 \\
\hline Infections & na & na & na & 5 & 23 & na & 35 & 21 \\
\hline Pneumonitis & na & 8 & na & 5 & na & 12 & 14.3 & 13.3 \\
\hline Hyperglycemia & na & na & na & 5 & na & na & 6.9 & 3.4 \\
\hline Atypical infection & na & na & na & na & na & 2 & na & na \\
\hline
\end{tabular}

na: not available. 
Table 3. Safety profile of sunitinib.

\begin{tabular}{|c|c|c|c|c|}
\hline \multirow[b]{2}{*}{ Grade } & \multicolumn{2}{|c|}{ Raymond et al. [7] } & \multicolumn{2}{|c|}{ Ito et al. [8] } \\
\hline & $\begin{array}{l}1-2 \\
(\%)\end{array}$ & $\begin{array}{l}3-4 \\
(\%)\end{array}$ & $\begin{array}{l}1-2 \\
(\%)\end{array}$ & $\begin{array}{l}3-4 \\
(\%)\end{array}$ \\
\hline Diarrhea & 30 & na & 83 & na \\
\hline Nausea & 30 & na & na & na \\
\hline Vomiting & 30 & na & na & na \\
\hline Asthenia & 30 & na & na & na \\
\hline Fatigue & na & na & 58 & na \\
\hline Headache & 26 & na & 58 & na \\
\hline Hypertension & 23 & 10 & 67 & na \\
\hline Hand foot syndrome & 23 & na & 67 & na \\
\hline Neutropenia & na & 12 & na & 50 \\
\hline Leukopenia & na & na & na & 17 \\
\hline Encephalitis & na & na & na & 33 \\
\hline Enterocolitis & na & na & na & 33 \\
\hline Hypothyroidism & 3 & na & na & na \\
\hline Lipase increase & na & na & na & 33 \\
\hline
\end{tabular}

na: not available.

Sunitinib is approved for the treatment of patients with unresectable or metastatic, progressive well differentiated pNEN. The most common adverse events reported with $37.5 \mathrm{mg} /$ day of sunitinib were diarrhea, fatigue, hypertension and mucocutaneus alterations [31]. A multicenter, open label, phase II study, conducted in 12 Japanese patients, with well differentiated pNEN, who received $37.5 \mathrm{mg} /$ day of sunitinib, demonstrated antitumor activity and a good safe profile. The most common adverse events were diarrhea (83\%), hand-foot syndrome and hypertension (67\%), fatigue and headache (58\%). Grade 3 neutropenia and leukopenia were reported in $50 \%$ and $17 \%$ of patients, respectively. Grade 4 events, such us herpes encephalitis with convulsion, increased lipase and enterocolitis were reported in 33\% of patients treated [32]. A multinational, randomized, double blind, placebo-controlled phase III trial was conducted in patients affected by advanced, well differentiated pancreatic neuroendocrine tumors. One hundred and seventy one patients were randomly assigned to receive placebo or sunitinib at dose of $37.5 \mathrm{mg} /$ day. The most frequents grade 1 or 2 adverse events were diarrhea, nausea, vomiting, asthenia and fatigue (30\% of patients treated). Palmar-plantar erythrodysesthesia and hypertension occurred respectively in $23 \%$ and $26 \%$ of treated patients with sunitinib. Hypothyroidism was reported in six patients treated with sunitinib. The most common grade 3 or 4 adverse events were neutropenia (12\%) and hypertension (10\%). One patient, treated with sunitinib, died during the study for grade 5 cardiac failure considered to be related to drug (Table 3) [30].

\section{Peptide receptor radionuclide therapy}

Peptide receptor radionuclide therapy (PRRT) is a systemic radiotherapy using radiolabeled synthetic and stable SSA $\left({ }^{90} \mathrm{Yttrium}\right.$ DOTATOC/DOTATATE and ${ }^{177}$ Lutetium DOTATOC/DOTATATE) that bind to SSTR expressed in NEN. The radiolabeled SSA are internalized into the cell, and the breakdown products of radiolabeled peptides are stored in lysosomes, thus enabling a long irradiation of tumor cells and causing radiation-induced cell death [33]. The two commonly used radionuclides for therapy are yttrium $(\mathrm{Y})$ and lutetium $(\mathrm{Lu}) .{ }^{90} \mathrm{Y}$, a high-energy beta emitter, is linked to octreotide constituting ${ }^{90} \mathrm{Y}$ DOTATOC, a high-energy radiopharmaceutical with good receptor affinity for the SSTR-2 peptide, tissue penetration of 7-8 mm and half-life of $64 \mathrm{~h} .{ }^{177} \mathrm{Lu}$ is a beta and gamma-emitting radionuclide. It has lower tissue penetration $(2 \mathrm{~mm})$ but a longer half-life $(160 \mathrm{~h})$ and a higher peptide receptor affinity for SSTR-2. Both ${ }^{90} \mathrm{Y}$ DOTATOC and ${ }^{177}$ Lu DOTATATE have similar efficacy. The reported objective response (OR) to ${ }^{90} \mathrm{Y}$ DOTATOC in different studies ranges from $20 \%$ to $28 \%$ for all neuroendocrine tumors and from $28 \%$ to $38 \%$ for GEP NEN [34]. Median PFS ranges from 17 to 29 months, and median overall survival (OS) from 22 to 37 months in advanced neuroendocrine tumors [35-38]. The reported OR to ${ }^{177} \mathrm{Lu}$ DOTATATE ranges from $39 \%$ to $60 \%$ in advanced pNEN [39-42]. Median OS and PFS reported in all NEN are 46 months and 33 months respectively [39]. Median PFS in pNEN ranges from 29 to 34 months [41,42] with a median OS reported of 53 months [42]. NETTER-1, the first and only phase-3 randomized controlled trial which evaluated the efficacy and safety of ${ }^{177}$ Lu DOTATATE in patients with metastatic or locally advanced midgut NEN, demonstrated an improved survival benefit when compared with octreotide LAR $60 \mathrm{mg}$ every 4 week [43]. PRRT is generally well tolerated but it is not completely free from side effects that can be acute or delayed. Acute side effects include nausea, vomiting and headache, usually mild and self-limiting. Other acute adverse events are fatigue, abdominal pain, asthenia and flushing. They usually occur in the early post injection phase (within $24 \mathrm{~h}$ ) and are generally transient [39]. Adverse events related to ${ }^{177}$ Lu DOTATATE treatment occurred in $86 \%$ patients of NETTER-1 study [43] and in $61 \%$ of patients of a phase Il study from Baum et al. [44] About $96 \%$ of patients experienced one or more adverse effects with ${ }^{90} \mathrm{Y}$ DOTATOC [36]. The most common events reported were gastrointestinal. After ${ }^{177} \mathrm{Lu}$ DOTATATE therapy nausea and vomiting were reported in $25 \%$ and $59 \%$ and in $10 \%$ and $47 \%$ of patients, in two different studies, respectively [39,43]. After ${ }^{90} \mathrm{Y}$ PRRT nausea was reported in 15\% [38] and 57\% of patients [36]. Grade 3-4 nausea was reported in $13 \%$ of patients [36]. Vomiting was reported in $8 \%$ [38] and $46 \%$ with a $10 \%$ of grade 3-4 [36]. A majority of these cases were attributable to amino acid infusions for renal protection and they resolved once the infusions were completed. Mild asthenia is generally experienced in the first week following therapy with both ${ }^{177} \mathrm{Lu}$ and ${ }^{90} \mathrm{Y}$. Asthenia and fatigue were reported in $40 \%$ of patients after ${ }^{177}$ Lu DOTATATE [43], and in $41 \%$ after ${ }^{90}$ Y DOTATOC [36]. Abdominal pain was reported in $10 \%$ and $26 \%$ related to ${ }^{177}$ Lu DOTATATE $[39,43]$ and in $5 \%$ and $21 \%$ related to ${ }^{90} \mathrm{Y}$ PRRT [36,38]. About $7 \%$ had grade 3-4 abdominal pain after ${ }^{90} \mathrm{Y}$ DOTATOC [36]. The incidence of diarrhea was similar after ${ }^{177}$ Lu DOTATATE and ${ }^{90} \mathrm{Y}$ DOTATOC, reported in $27 \%$ and $29 \%$, respectively $[36,43]$. Peripheral edema was reported in $14 \%$ of patients with no cases of grade 3-4 after ${ }^{177}$ Lu DOTATATE [43]. After ${ }^{90} Y$ DOTATOC it was reported in $9 \%$ of patients with $1 \%$ of grade 3 toxicity [36]. Mild and temporary alopecia was observed with ${ }^{177}$ Lu DOTATATE in $11 \%$ of patients in NETTER-1 study [43] and in $62 \%$ of patients in a series of over 500 patients from Kwekkeboom et al. [39]. Hormonal crisis has been described as acute effects. A very small minority (1\%) of patients after ${ }^{177} \mathrm{Lu}$ DOTATATE develops a carcinoid crisis usually within $48 \mathrm{~h}$ of the first infusion [45]. Furthermore, hormone related syndrome have been reported in $1 \%$, for both ${ }^{90} \mathrm{Y}$ DOTATATE [38] and ${ }^{177} \mathrm{Lu}$ DOTATATE [39]. Flushing, reported immediately or within 6-12 h, was reported in 5-22\% after ${ }^{90} \mathrm{Y}$ PRRT therapy [36,38]. Delayed side 
effects include myelosuppression and impaired renal function. Late hematological toxicity in the form of acute leukemia (AL) and myelodysplastic syndrome (MDS) has also been reported. Myelosuppression is due to the cumulative effects, by contrast renal function do not show any correlation with cumulative activity received [44]. Therefore, the kidneys appear to be the critical doselimiting organs. Myelosuppression often occurs after three doses (4-6 weeks) and is usually mild and transient. In a phase II study from Sansovini et al. a transient grade 1-2 leukopenia in 19\% of patients, a grade 1-2 thrombocytopenia and anemia in $17 \%$ and $32 \%$ of patients after ${ }^{177}$ Lu DOTATE were reported. No hematological toxicity of grade 3-4 was reported [41]. In NETTER-1 study thrombocytopenia and lymphopenia were the most common alterations, reported respectively in $25 \%$ and $18 \%$ of patients; grade 3-4 lymphopenia was reported in $9 \%$ of patients [43]; however clinical impact of leukopenia and lymphopenia is of doubt importance since opportunistic infection are not reported with PRRT [46]. In the phase I-II study from Bodei et al. after ${ }^{177} \mathrm{Lu}$ DOTATATE grade 1-2 anemia, leukopenia and thrombocytopenia in $78 \%, 61 \%$ and $27 \%$ were reported respectively. Only one (2\%) patient had transient grade 3 leukopenia and thrombocytopenia [40]. Radiopeptides are reabsorbed in the proximal tubules and can accumulate in the renal interstitium inducing inflammation and fibrosis. With advances in expertise and knowledge about PRRT and renal protection, cases of severe end stage renal failure are extremely rare. Renal toxicity is generally limited by renal protection obtained with amino acid infusion, competing with the site of tubular reabsorption of the drug, reducing the recirculation of radionuclides and thus limiting renal exposure. ${ }^{90} \mathrm{Y}$ PRRT is associated with significantly higher nephrotoxicity. Because of its higher energy and longer penetration range, ${ }^{90} \mathrm{Y}$ irradiates renal interstitium and glomeruli more extensive than ${ }^{177} \mathrm{Lu}$. Despite renal protection a median annual decline of about $7 \%$ per year in creatinine clearance for ${ }^{90} \mathrm{Y}$ DOTATOC have been reported so that the loss of renal function may become clinically evident from 1 to 5 years after therapy in some patients [35]. In a further study severe permanent renal failure (grade 4-5) occurred in 9\% of patients after ${ }^{90}$ Y DOTATOC therapy [47]. Valkema et al. reported more than $15 \%$ per year decline in clearance creatinine in $15 \%$ of patients and end renal stage in $3 \%$ of patients treated with ${ }^{90} \mathrm{Y}$ DOTATOC [48]. In the NETTER-1 study, no evidence of renal toxic effects was reported among patients in the ${ }^{177}$ Lu DOTATATE group (median duration follow-up of 14 months). No cases of major, grade 3-4, renal toxicity related to ${ }^{177} \mathrm{Lu}$ DOTATATE were reported in previous retrospective and prospective studies (median duration followup ranges from 18 to 58 months) [39,40,49,50], in particular in the treatment of patients without risk factors [41]. A range from $2 \%$ to $4 \%$ per year of annual decline in creatinine clearance also for ${ }^{177}$ Lu DOTATATE was reported [35,42]. Preexisting risk factors such as long-standing and poorly controlled diabetes and hypertension are considered risk factors for delayed renal toxicity and contribute to the loss of renal function [51]. However, other intrinsic biological factors, probably of genetic origin, cannot be excluded as involved in the development of renal toxicity [49]. In different studies MDS and $A L$ have been reported in about $1-2 \%$ of patients receiving PRRT. Incidence of MDS and AL after both ${ }^{177}$ Lu-PRRT and ${ }^{90} \mathrm{Y}$-PRRT is similar. MDS and AL usually develop several years after completed therapy so their occurrence may be underestimated because of the short follow-up of the patients. Furthermore, the causal relationship with PRRT may be questioned, because of previous treatments, such as chemotherapy with alkylating agents or radiotherapy. No significant difference in the development of MDS or AL was reported from Bodei et al. [49] when ${ }^{90} \mathrm{Y}$-based and

${ }^{177}$ Lu-based therapies were compared. In NETTER-1 study only one patient $(0.9 \%)$ developed MDS probably related to the PRRT [43]. Kwekkeboom et al. [39] reported in a population of 504 patients, four cases (0.7\%) of MDS diagnosed 2-3 year after the last treatment with ${ }^{177}$ Lu DOTATATE, three cases probably treatment related (no previous treatment with alkylating agents). One patients (1.7\%) developed MDS 2 years after the start of PRRT with ${ }^{90} \mathrm{Y}$ DOTATOC [48]. Similarly, Pfeifer et al. [37] observed one proven case $(1.8 \%)$ and one case suspicious for MDS related to ${ }^{90} \mathrm{Y}$ DOTATOC treatment. Both patients had previously been treated with alkylating agents, which could have contributed to the development of these outcomes. A theoretical risk of hepatotoxicity and liver failure is in patients with high burden of liver metastasis. No evidence of liver toxicity was reported in NETTER-1 study [43]. In the phase II study from Baum et al. a significant correlation of serum glutamic oxaloacetic transaminase (SGOT), serum glutamic-pyruvic transaminase (SGPT) and alkaline phosphatase (SAP) with the administered radioactivity $\left({ }^{177}\right.$ Lu DOTATOC) was observed. According to authors these correlations may indicate persistent or new growth of hepatic metastases causing a rise of hepatic enzymes, but a minor liver damage related to PRRT cannot be excluded [44]. In previous study, serious liver toxicity was reported in $0.5 \%$ of patients treated with ${ }^{177}$ Lu DOTATE. One patient died of hepatic failure, but according to authors the liver failure was more likely tumor growth-related than radiation induced [39]. Similarly ${ }^{90} \mathrm{Y}$ DOTATOC treated patients showed a low liver toxicity and grade 3 toxicity was observed in very few patients (1.7\%) [48]. PRRT safety is summarized in Table 4.

\section{Chemotherapy}

According to ENETS guidelines, cytotoxic chemotherapy is indicated in G3 NEN, in progressive or advanced pNEN, and even in case of rapidly progressive disease and/or after failure of other therapies, or negativity of SSTR imaging [4,52]. In advanced progressive G1-G2 NEN, alkylating agents such as streptozocin (STZ) and temozolomide are used. These compounds proved to be particularly active in PNEN [53]. STZ is a nitrosourea alkylating agent. Since the eighties, schedules involving STZ have been used as standard of cure for well and moderately differentiated pNEN. Combination with 5-florouracil (5-FU) [54], with doxorubicin [55], with 5-FU and doxorubicin [56], with 5-FU and cisplatin (CIS) [57], with capecitabine (CAP) and with CAP and CIS [58] have been tried. All of these combinations were associated with partial response rates between $25 \%$ and $39 \%$ and median OS between approximately 2 and 4 years. STZ plus 5 -FU is an established therapy in G1-G2 pNEN [4]. The most common adverse events reported in a retrospective study were kidney toxicity, nausea and fatigue. No hematological adverse events were reported. The majority of adverse events were of grade 1 [54]. STZ plus doxorubicin, an anthracycline neoplastic agent, is an alternative option but the applicability of this drug is limited by the risk of cardiotoxicity [4]. Anthracyclines-induced 


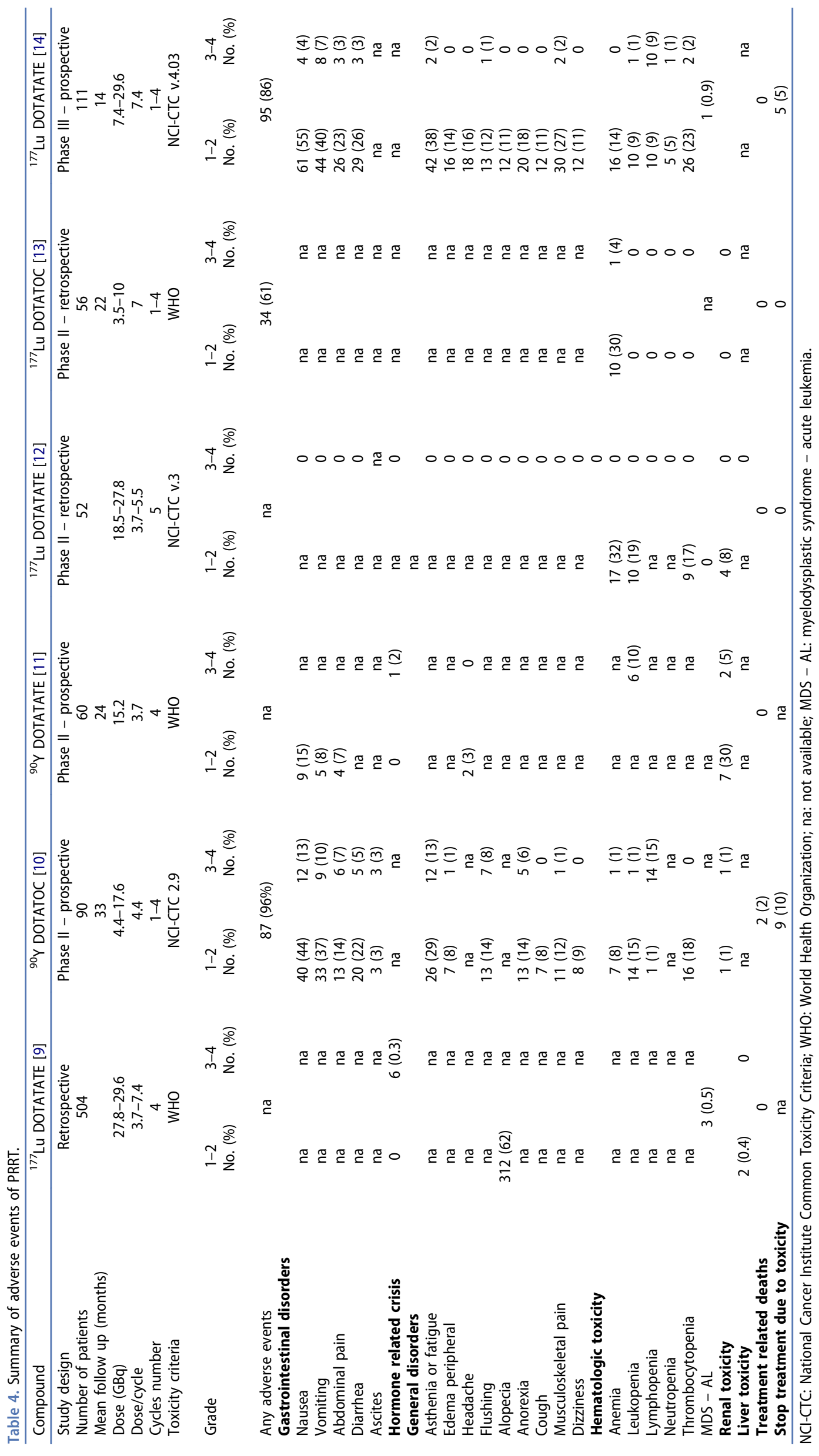


cardiotoxicity has been widely studied in other neoplasm types. Despite long-term research anthracycline cardiotoxicity mechanisms are not fully known. Multiple complex mechanisms are involved in anthracycline cardiotoxicity which may manifest either as an initial acute effect with pericarditis and arrhythmias, or a later cumulative dose dependent cardiomyopathy [59]. The incidence of cardiomyopathy at cumulative dose of $400 \mathrm{mg} / \mathrm{mq}$ is $5 \%$ and reaches up to $26 \%$ of patients at a cumulative dose of $550 \mathrm{mg} / \mathrm{mq}$ [60]. Until now prospective randomized trials to evaluate cytotoxic chemotherapy in patients with advanced NEN have been scarcely taken in account. The NET01, a multicenter randomized prospective study, is the first trial formally applying RECIST criteria in this setting. The NET01 compared two different schedules (CAPSTZ-CIS vs. CAP-STZ). The efficacy of the two schedules was very similar. CAP-STZ schedule was better tolerated than CAPSTZ-CIS, furthermore addition of CIS was not associated with any definite improvement in response rate or survival. Considering all grade adverse events, the most common was fatigue $(72 \%$ of patients in CAP-STZ and $80 \%$ of patients in CAP-STZ-CIS). Nausea/vomiting and diarrhea were experienced by $63 \%$ and $51 \%$ of patients in CAP-STZ schedule, and $70 \%$ and $48 \%$ of CAP-STZ-CIS schedule respectively. Grade $\geq 3$ toxicity was experienced by $44 \%$ CAP-STZ patients compared to $68 \%$ CAP-STZ-CIS patients. Combining both arms the most common grade 3-4 toxicities were gastrointestinal disorders (diarrhea, nausea, vomiting and mucositis) in 18\% of patients; fatigue and thromboembolic events were experienced in $12 \%$ and $10 \%$ respectively. Grade 3 neutropenia was experienced by $8 \%$ of patients in CAP-STZ-CIS schedule, whereas no grade 3 neutropenia was reported in CAP-STZ treated patients. Grade $\geq 3$ renal toxicity was reported in $2 \%$ and in $10 \%$ in CAP-STZ and CAP-STZ-CIS respectively. There were no grade 5 treatment related adverse effects [58]. STZ remains the standard schedule but recently temozolomide-based schedule has rapidly gained in wide clinical application with an impressive response rate of $70 \%$ [53]. Temozolomide, a new second generation alkylating agent, penetrates the blood-brain barrier, can be taken orally, and is better tolerated than other alkylating agents. Temozolomide as monotherapy in NEN has been evaluated only in retrospective analysis demonstrating antitumoral activity with acceptable toxicity. Toxicities were mainly hematologic. Considering grade 3 toxicity, thrombocytopenia was the most common side effect (14\% of patients) but no patients had bleeding due to low platelets. Neutropenia grade 4 was reported in $3 \%$ of patients but none had febrile neutropenia and opportunistic infections. Fatigue and nausea were the most common adverse effects reported, but they were mild in the majority of patients $(23 \%$ and $29 \%$ grade 1 respectively). A rate of $6 \%$ of patients had grade 3-4 fatigue [61]. Strosberg et al. investigated temozolomide in combination with CAP in the first line treatment of metastatic well or moderately differentiated pNEN in a retrospective nonrandomized study. They observed an objective response rate of $70 \%$. Toxicity rates were considerably lower than those observed with STZ-based schedules. Grade 1-2 events were limited and there was a $12 \%$ rate of grade 3-4 toxicities associated with this schedule. The most common minor adverse events were fatigue (12\%), nausea (16\%), myelosuppression (43\%) and hand-foot skin reaction (23\%). Grade 3 adverse effects reported were elevated AST (3\%), fatigue (3\%), whereas grade 4 were leukopenia (3\%) and thrombocytopenia (3\%) [53]. Fine et al. also evaluated safety of CAP plus temozolomide in a retrospective study conducted on 18 patients with metastatic well differentiated NEN. No grade 4 toxicity was reported. Grade 3 thrombocytopenia was reported in $11 \%$ of patients. The most common toxicity was of grade 1-2 with lymphopenia, neutropenia and hand-foot syndrome reported in $50 \%, 44 \%$ and $5.5 \%$ of patients, respectively. No cases of opportunistic infections, sepsis, neutropenic fevers and deaths related to treatment were reported [62]. Saif et al., although the small sample size of seven patients, reported similar results in a retrospective study of CAP plus temozolomide schedule in the treatment of metastatic pNEN [63]. Temozolomide treatment is costly and currently not approved for the treatment of pNEN yet. Dacarbazine (DTIC), another alkylating agent sharing the active metabolite metozolomide with temozolomide, is broadly available at a low cost. In a phase II study of 50 patients with advanced pNEN, the response rate to DTIC was $34 \%$ with a median OS of 19.3 months [64]. The efficacy and safety of a lower dose DTIC schedule in patients with progressive advanced NEN has recently been evaluated in a retrospective study. Mueller et al. reported a response rate of $66 \%$ (partial remission and disease stabilization). In contrast to previous reports with more dose intense DTIC schedules, a good safety profile was demonstrated. Adverse effects were documented in $57 \%$ of patients and they were usually mild and transient. The most common adverse effects were gastrointestinal, such as nausea, vomiting and diarrhea, reported in $31 \%, 17 \%$ and $15 \%$ respectively. However, the majority of patients had grade 1-2 gastrointestinal effects. Only $1 \%$ of patients reported grade 3 vomiting. Grade 1 elevated liver enzymes, fatigue and weight loss were reported in 14\%,5\% and $3 \%$ respectively. In contrast to previous study, no hematologic toxicity of grade 3-4 was reported. The most common grade 1-2 hematologic adverse effects were anemia, leukopenia and thrombocytopenia reported in 5\%, $8 \%$ and $3 \%$ respectively [65]. Platinum-based schedules are used for G3 NEN. No phase III studies have been performed for G3 NEN. CIS combined with etoposide is the first line recommended chemotherapy [4]. The side effects most frequently associated with platinum include myelosuppression (leukopenia, thrombocytopenia and anemia), gastrointestinal (anorexia, nausea, vomiting and diarrhea), nephrotoxicity (renal failure, hyperuricemia) and ototoxicity (hearing impairment). Neuropathy, in particular peripheral sensory neurotoxicity, is also common. More rare adverse events include visual impairment, seizures, arrhythmias, acute ischemic vascular events, glucose intolerance and pancreatitis. Myelosuppression is the dose-limiting toxicity of platinum based schedules [52]. Carboplatin has been tested as an alternative to CIS. According to Nordic NEC study, carboplatin could replace CIS, as the two compounds were comparable in efficacy, PFS and survival [66]. Carboplatin plus etoposide seems have milder adverse events than CIS plus etoposide. The incidence of thrombocytopenia is higher with 
carboplatin-based chemotherapy than with CIS, while no difference in the incidence of grade 3-4 anemia and neutropenia was observed. In a retrospective case series of 19 patients treated with carboplatin plus etoposide grade 3-4 neutropenia and leukopenia were the most common toxicities, reported in $78 \%$ and $73 \%$ respectively. Grade 3-4 anemia and thrombocytopenia were reported in $21.1 \%$ and $26.3 \%$ respectively. Severe hepatotoxicity, in term of elevation of transaminase was reported in $15.8 \%$. No increase of bilirubin was observed. Severe anorexia and nausea were reported in $10.5 \%$ and $5.8 \%$ respectively [67]. Although hematological toxicities reported with carboplatin is similar, the other toxicities of carboplatin are generally milder and better tolerated than those of CIS. The incidence of grade 3-4 elevation of bilirubin and grade 3-4 nausea was lower than incidence reported with CIS (19\% and 33\% respectively). Nausea and vomiting are usually less severe and shorter in duration. No differences in the incidence of renal impairment was observed [68]. Etoposide is a semisynthetic derivative of podophyllotoxin available for intravenous use. Myelosuppression is the dose limiting toxicity of etoposide. Nausea and vomiting are the major gastrointestinal toxicities. Other gastrointestinal toxicities include anorexia, stomatitis and diarrhea. Alopecia is usually reversible and it has been observed in up $66 \%$ of patients. Transient hypotension and anaphylactic type reactions have been reported to occur after administration of etoposide [52]. Alternative schedule substituting irinotecan for etoposide is recommended as first-line therapy [52]. Irinotecan is a DNA topoisomerase I inhibitor. Hematological toxicities (neutropenia, anemia ad thrombocytopenia) are the most frequent dose-limiting toxicities of irinotecan. Irinotecan can cause acute diarrhea (immediately after drug administration) and delayed diarrhea (occurring more than $24 \mathrm{~h}$ after drug administration). Acute diarrhea is caused by acute cholinergic properties and can be accompanied by a transient acute cholinergic syndrome, characterized by abdominal cramps, vasodilatation, rhinitis, sweating, dizziness, visual disturbances, miosis, lacrimation and increased salivation. Other side effects include alopecia, asthenia, increase in serum levels of ALT, AST, SAP, bilirubin, amylase and lipase [52]. Kulke et al. reported in a phase II trial of irinotecan and CIS mild toxicity including myelosuppression, nausea and diarrhea. Myelosuppression was the most common treatment related toxicity. Grade 3-4 neutropenia was reported in 39\% of patients. Grade 3 nausea and vomiting was reported in $21 \%$ of patients. Diarrhea was reported in $66 \%$ of patients but only 1 patient had grade 3-4 (5.6\%) Other grade 3-4 adverse advents were fatigue (5.6\%), abdominal pain (5.6\%), anorexia (5.6\%) and constipation (5.6\%) [69]. Similarly, hematological toxicity was the most common reported in a retrospective series of patients treated with irinotecan and CIS from $\mathrm{Lu}$ et al. Neutropenia was reported in $69 \%$ of patients, $9(56.3 \%)$ of them of grade 3-4 [70]. Similar results were reported from Nakano et al. In addition, they reported a significative incidence of grade 3-4 of infections (14\%) and of electrolyte imbalance $(20 \%)$ including hyponatremia and hyperkalemia [71]. FOLFOX (folinic acid-fluorouracil-oxaliplatin) and FOLFIRI (folinic acid-fluorouracil-irinotecan) schedules are the second line systemic therapy option [4,52]. Sensory neuropathy is the dose-limiting toxicity of oxaliplatin, a third generation platinum analogue while myelosuppression and nephrotoxicity are uncommon. Most common adverse events related to FOLFOX schedule were asthenia (80\%), with a $10 \%$ of grade $3-4$, nausea and vomiting (40\%) with $10 \%$ of grade $3-4$, and diarrhea (35\%) with a $5 \%$ of grade $3-4$. Severe anemia, thrombocytopenia and neutropenia were reported in $10 \%, 10 \%$ and $20 \%$ respectively. Symptomatic neurotoxicity with any severity was reported in $80 \%$ of patients. One patient had grade 3 neurotoxicity [72]. Main adverse events reported with FOLFIRI were neutropenia and diarrhea (grade 3-4, 32\%) in patients with neuroendocrine carcinomas. Among them 1 patient (5\%) experienced grade 3 neutropenia and $3(16 \%)$ patients experienced grade 3 diarrhea. Grade 4 neutropenia was reported in $3(16 \%)$ patients [73]. Temozolomide is an option for palliative treatment in patients with neuroendocrine carcinoma although prospective studies to assess the activity of temozolomide in this setting are necessary. Regarding safety of temozolomide in this setting, Olsen et al. reported similar results to those reported in well differentiated NEN [74]. Detailed adverse events incidence of main chemotherapeutic schedules is summarized in Table 5.

\section{Conclusions}

Biotherapy with SSA, targeted therapies with mTOR and tyrosine kinase inhibitors, PRRT and chemotherapy represent well established systemic therapies for NEN. The biology of NEN explains the effectiveness of these compounds acting, with the exception of chemotherapy, on the basis of specific molecular targets. The indolent clinical course of the well differentiated G1-G2 NEN makes possible to stabilize tumor growth by chronic therapies over a long period of time. If these therapeutic options proved to be effective, on the other hand, they seem also safe, manageable and well tolerated. This is true not only for SSA but also for targeted therapies and PRRT. In particular, octreotide and lanreotide have to be managed for gastrointestinal abnormalities and cholelithiasis while pasireotide also for hyperglycemia. However, treatment discontinuation as well as dose adjustment are rarely required. Targeted therapies with everolimus and sunitinib are quite more toxic than SSA but, even in these cases, adverse events can be for the most managed and prevented, sometimes decreasing the dose or temporarily interrupting the administration and rarely discontinuing the drug. ${ }^{177}$ Lutetium PRRT has been recently investigated in a phase III clinical trial, revealing a low rate of serious adverse events. However, renal and hematologic function should be long-time monitored because of the possible occurrence of renal insufficiency and myelodysplastic syndrome. Chemotherapy is usually considered for highly proliferating poorly differentiated tumors or for advanced well differentiated tumors, progressive after previous therapeutic lines. For this reason, cumulative toxicities and poor performance status of these patients, could in part explain the higher rate of chemotherapy-related serious adverse events. However, grade 3-4 hematological adverse events were more frequently seen in patients treated with platinum-based schedule. 


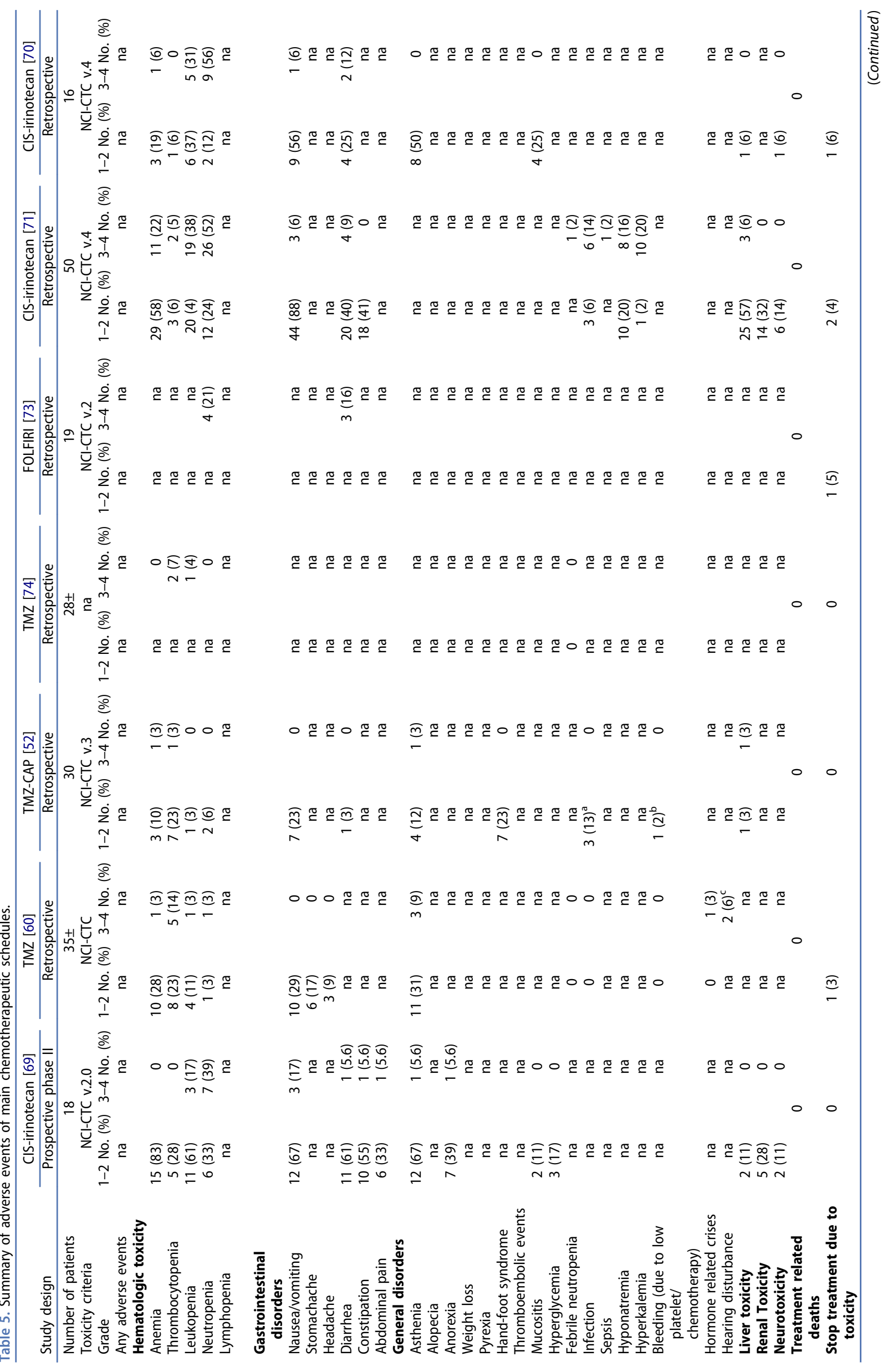




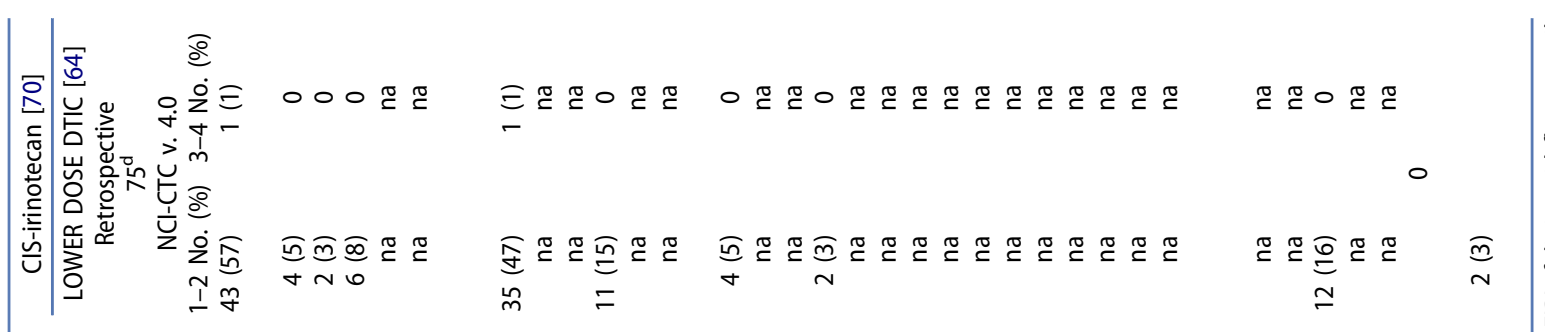

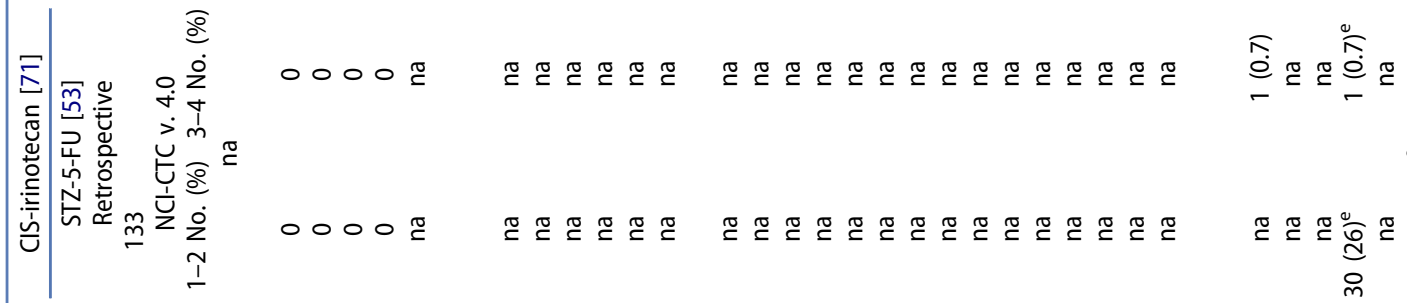

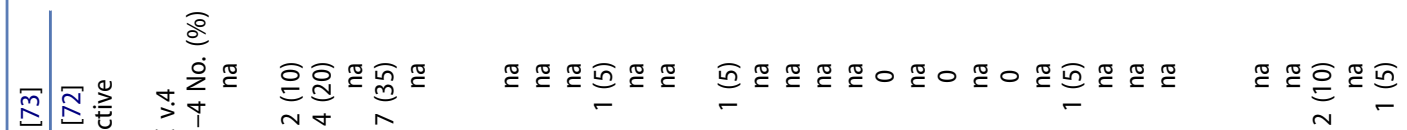

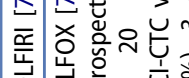

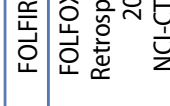

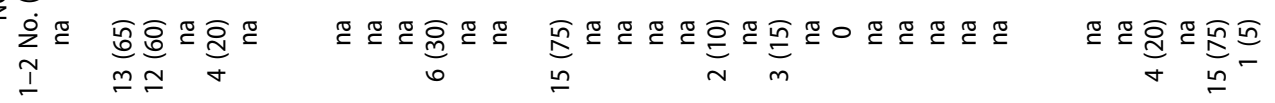

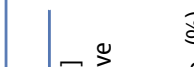

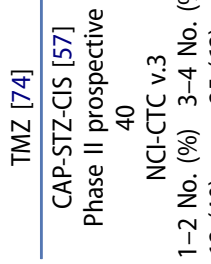

$\dot{s}$

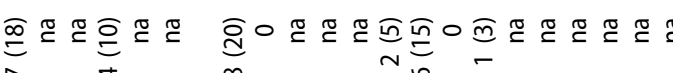

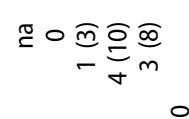

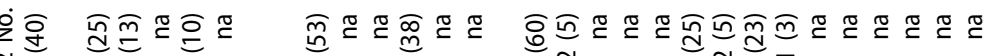

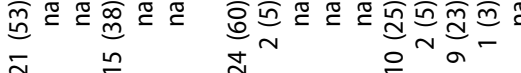

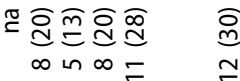

बำ

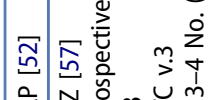

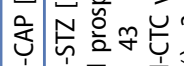

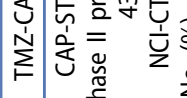

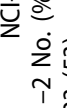

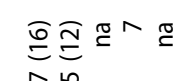

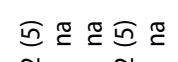

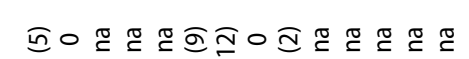

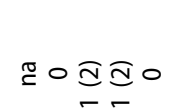

1 वे

要

实

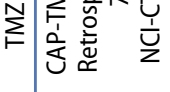

ì

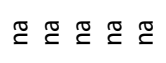

잉

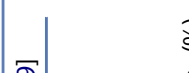

总

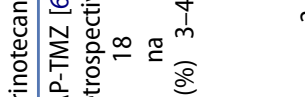

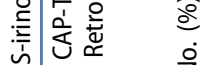

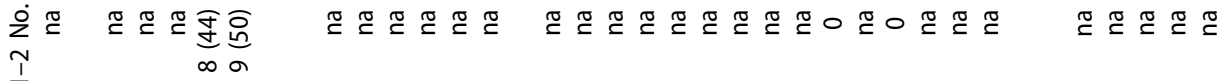

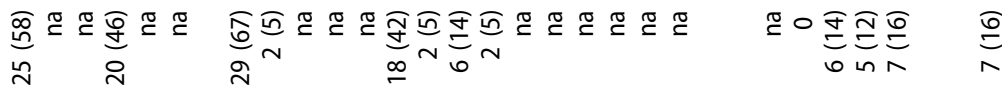

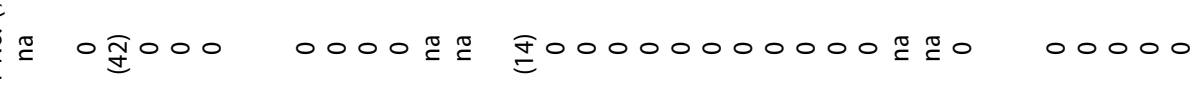

I
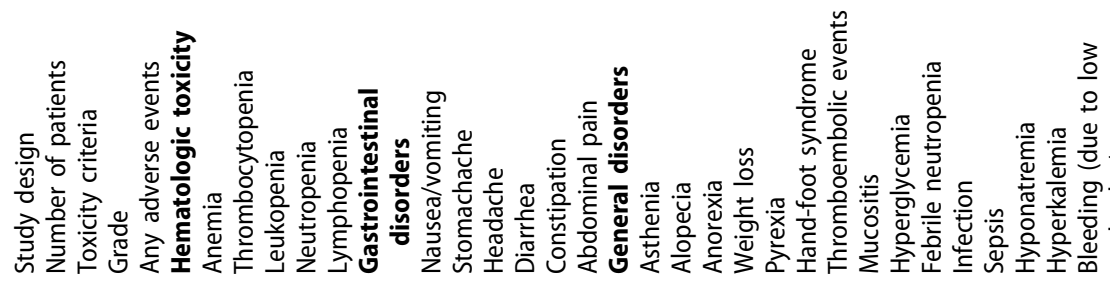

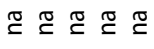

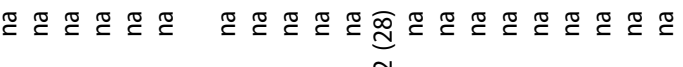

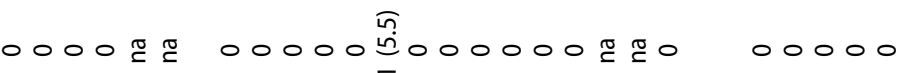




\section{Funding}

This paper has not been funded.

\section{Declaration of interest}

A Colao has been principal investigator of research studies from Novartis, Ipsen, Pfizer and Lilly, has received research grants from Ferring, Lilly, Ipsen, Merck-Serono, Novartis, Novo-Nordisk and Pfizer, has been occasional consultant for Novartis, Ipsen and Pfizer, and has received fees and honoraria from Ipsen, Novartis, and Pfizer. A Faggiano has received support for research from Novartis and Ipsen, has been occasional consultant from Novartis, Ipsen and Pfizer, and has received fees from Ipsen, Novartis and Pfizer. Other authors have no conflict of interest to declare. The authors have no other relevant affiliations or financial involvement with any organization or entity with a financial interest in or financial conflict with the subject matter or materials discussed in the manuscript apart from those disclosed.

\section{References}

Papers of special note have been highlighted as either of interest $(\cdot)$ or of considerable interest $(\cdot \bullet)$ to readers.

1. Leoncini E, Boffetta P, Shafir M, et al. Increased incidence trend of low-grade and high-grade neuroendocrine neoplasms. Endocrine. 2017 Mar 16. Epub ahead of print.

2. Bosman FT, World Health Organization. International Agency for Research on Cancer. WHO classification of tumours of the digestive system. 4th ed. Lyon: International Agency for Research on Cancer; 2010.

3. Garcia-Carbonero R, Sorbye $H$, Baudin E, et al. ENETS consensus guidelines for high-grade gastroenteropancreatic neuroendocrine tumors and neuroendocrine carcinomas. Neuroendocrinology. 2016;103(2):186-194.

4. Pavel $M, O$ 'Toole $D$, Costa $F$, et al. ENETS consensus guidelines update for the management of distant metastatic disease of intestinal, pancreatic, bronchial neuroendocrine neoplasms (NEN) and NEN of unknown primary site. Neuroendocrinology. 2016;103(2):172-185.

5. Rinke A, Muller HH, Schade-Brittinger $\mathrm{C}$, et al. Placebo-controlled, double-blind, prospective, randomized study on the effect of octreotide LAR in the control of tumor growth in patients with metastatic neuroendocrine midgut tumors: a report from the PROMID Study Group. J Clin Oncol. 2009 Oct 1;27(28):4656-4663.

.- The first study to show an antiproliferative effect with a somatostatine analog.

6. Oberg K, Lamberts SW. Somatostatin analogues in acromegaly and gastroenteropancreatic neuroendocrine tumours: past, present and future. Endocr Relat Cancer. 2016 Dec;23(12):R551-R66.

7. Dowling RH, Hussaini SH, Murphy GM, et al. Gallstones during octreotide therapy. Metabolism. 1992 Sep;41(9 Suppl 2):22-33.

8. Ogmen BPB, Cuhaci N, Aydin C, et al. Lanreotide-induced bradycardia and supraventricular extrasystoles. Endocr Abstr. 2015;35: P916.

9. Colao A, Auriemma RS, Galdiero M, et al. Impact of somatostatin analogs versus surgery on glucose metabolism in acromegaly: results of a 5-year observational, open, prospective study. J Clin Endocrinol Metab. 2009 Feb;94(2):528-537.

10. Caplin ME, Pavel M, Cwikla JB, et al. Lanreotide in metastatic enteropancreatic neuroendocrine tumors. N Engl J Med. 2014 Jul 17;371(3):224-233.

. The CLARINET study, a phase III trial, provided important information on the use of lanreotide in clinical practice demonstrating antiproliferative activity and the favorable tolerability and safety.

11. Chadha MK, Lombardo J, Mashtare T, et al. High-dose octreotide acetate for management of gastroenteropancreatic neuroendocrine tumors. Anticancer Res. 2009 Oct;29(10):4127-4130.

12. Ruszniewski $P$, Ish-Shalom $S$, Wymenga $M$, et al. Rapid and sustained relief from the symptoms of carcinoid syndrome: results from an open 6-month study of the 28-day prolonged-release formulation of lanreotide. Neuroendocrinology. 2004;80(4):244-251.

13. Broder MS, Beenhouwer D, Strosberg JR, et al. Gastrointestinal neuroendocrine tumors treated with high dose octreotide-LAR: a systematic literature review. World J Gastroenterol. 2015 Feb 14;21 (6):1945-1955.

14. Ferolla P, Faggiano A, Grimaldi F, et al. Shortened interval of longacting octreotide administration is effective in patients with welldifferentiated neuroendocrine carcinomas in progression on standard doses. J Endocrinol Invest. 2012 Mar;35(3):326-331.

15. Wolin EM, Hu K, Hughes G, et al. Safety, tolerability, pharmacokinetics, and pharmacodynamics of a long-acting release (LAR) formulation of pasireotide (SOM230) in patients with gastroenteropancreatic neuroendocrine tumors: results from a randomized, multicenter, open-label, phase I study. Cancer Chemother Pharmacol. 2013 Aug;72(2):387-395.

16. Wolin EM, Jarzab B, Eriksson B, et al. Phase III study of pasireotide long-acting release in patients with metastatic neuroendocrine tumors and carcinoid symptoms refractory to available somatostatin analogues. Drug Des Devel Ther. 2015;9:5075-5086.

17. Barbieri F, Albertelli M, Grillo F, et al. Neuroendocrine tumors: insights into innovative therapeutic options and rational development of targeted therapies. Drug Discov Today. 2014 Apr;19(4):458-468.

18. Watanabe R, Wei L, Huang J. mTOR signaling, function, novel inhibitors, and therapeutic targets. J Nuclear Medicine. 2011 Apr;52(4):497-500.

19. Bousquet C, Lasfargues C, Chalabi M, et al. Clinical review: current scientific rationale for the use of somatostatin analogs and mTOR inhibitors in neuroendocrine tumor therapy. J Clin Endocrinol Metab. 2012 Mar;97(3):727-737.

20. Kahan BD. The potential role of rapamycin in pediatric transplantation as observed from adult studies. Pediatr Transplant. 1999 Aug;3 (3):175-180

21. Eisen $H J$, Tuzcu EM, Dorent $R$, et al. Everolimus for the prevention of allograft rejection and vasculopathy in cardiac-transplant recipients. N Engl J Med. 2003 Aug 28;349(9):847-858.

22. O'Donnell A, Faivre S, Burris HA 3rd, et al. Phase I pharmacokinetic and pharmacodynamic study of the oral mammalian target of rapamycin inhibitor everolimus in patients with advanced solid tumors. J Clin Oncol. 2008 Apr 1;26(10):1588-1595.

23. Yao JC, Phan AT, Chang DZ, et al. Efficacy of RAD001 (everolimus) and octreotide LAR in advanced low- to intermediate-grade neuroendocrine tumors: results of a phase II study. J Clin Oncol. 2008 Sep 10;26(26):4311-4318.

24. Yao JC, Lombard-Bohas C, Baudin E, et al. Daily oral everolimus activity in patients with metastatic pancreatic neuroendocrine tumors after failure of cytotoxic chemotherapy: a phase II trial. J Clin Oncol. 2010 Jan 1;28(1):69-76.

25. Pavel ME, Hainsworth JD, Baudin E, et al. Everolimus plus octreotide long-acting repeatable for the treatment of advanced neuroendocrine tumours associated with carcinoid syndrome (RADIANT-2): a randomised, placebo-controlled, phase 3 study. Lancet. 2011 Dec 10;378(9808):2005-2012.

26. Yao JC, Shah $\mathrm{MH}$, Ito $\mathrm{T}$, et al. Everolimus for advanced pancreatic neuroendocrine tumors. N Engl J Med. 2011 Feb 10;364(6):514-523.

27. Yao JC, Fazio N, Singh $S$, et al. Everolimus for the treatment of advanced, non-functional neuroendocrine tumours of the lung or gastrointestinal tract (RADIANT-4): a randomised, placebo-controlled, phase 3 study. Lancet. 2016 Mar 5;387(10022):968-977.

- The last published RADIANT trials evaluating efficacy and safety of everolimus.

28. Faggiano A, Malandrino P, Modica R, et al. Efficacy and safety of everolimus in extrapancreatic neuroendocrine tumor: a comprehensive review of literature. Oncologist. 2016 Jul;21(7):875-886.

29. Yao VJ, Ozawa MG, Varner AS, et al. Antiangiogenic therapy decreases integrin expression in normalized tumor blood vessels. Cancer Res. 2006 Mar 1;66(5):2639-2649.

30. Raymond E, Dahan L, Raoul JL, et al. Sunitinib malate for the treatment of pancreatic neuroendocrine tumors. $N$ Engl J Med. 2011 Feb 10;364(6):501-513. 
31. Capdevila J, Sevilla I, Alonso V, et al. Evaluation of the efficacy and safety of lanreotide in combination with targeted therapies in patients with neuroendocrine tumours in clinical practice: a retrospective cross-sectional analysis. BMC Cancer. 2015 Jul;04(15):495.

32. Ito T, Okusaka T, Nishida T, et al. Phase II study of sunitinib in Japanese patients with unresectable or metastatic, well-differentiated pancreatic neuroendocrine tumor. Invest New Drugs. 2013 Oct;31(5):1265-1274.

33. Kwekkeboom DJ, Krenning EP. Peptide receptor radionuclide therapy in the treatment of neuroendocrine tumors. Hematol Oncol Clin North Am. 2016 Feb;30(1):179-191.

34. Nisa L, Savelli G, Giubbini R. Yttrium-90 DOTATOC therapy in GEPNET and other SST2 expressing tumors: a selected review. Ann Nucl Med. 2011 Feb;25(2):75-85.

35. Valkema R, Pauwels SA, Kvols LK, et al. Long-term follow-up of renal function after peptide receptor radiation therapy with $(90) \mathrm{Y}$ DOTA(0),Tyr(3)-octreotide and (177)Lu-DOTA(0), Tyr(3)-octreotate. J Nuclear Medicine. 2005 Jan;46(Suppl 1):83S-91S.

36. Bushnell DL Jr., O'Dorisio TM, O'Dorisio MS, et al. 90Y-edotreotide for metastatic carcinoid refractory to octreotide. J Clin Oncol. 2010 Apr 1;28(10):1652-1659.

37. Pfeifer AK, Gregersen T, Gronbaek H, et al. Peptide receptor radionuclide therapy with Y-DOTATOC and (177)Lu-DOTATOC in advanced neuroendocrine tumors: results from a Danish cohort treated in Switzerland. Neuroendocrinology. 2011;93(3):189-196.

38. Cwikla JB, Sankowski A, Seklecka N, et al. Efficacy of radionuclide treatment DOTATATE Y-90 in patients with progressive metastatic gastroenteropancreatic neuroendocrine carcinomas (GEP-NETs): a phase II study. Ann Oncology. 2010 Apr;21(4):787-794.

39. Kwekkeboom DJ, De Herder WW, Kam BL, et al. Treatment with the radiolabeled somatostatin analog [177 Lu-DOTA 0,Tyr3]octreotate: toxicity, efficacy, and survival. J Clin Oncol. 2008 May 1;26 (13):2124-2130.

40. Bodei L, Cremonesi M, Grana CM, et al. Peptide receptor radionuclide therapy with $(1,7,7)$ Lu-DOTATATE: the IEO phase I-II study. Eur J Nucl Med Mol Imaging. 2011 Dec;38(12):2125-2135.

41. Sansovini M, Severi S, Ambrosetti A, et al. Treatment with the radiolabelled somatostatin analog Lu-DOTATATE for advanced pancreatic neuroendocrine tumors. Neuroendocrinology. 2013;97(4):347-354.

42. Ezziddin S, Khalaf F, Vanezi M, et al. Outcome of peptide receptor radionuclide therapy with $177 \mathrm{Lu}$-octreotate in advanced grade $1 / 2$ pancreatic neuroendocrine tumours. Eur J Nucl Med Mol Imaging. 2014 May;41(5):925-933.

43. Strosberg J, El-Haddad G, Wolin E, et al. Phase 3 trial of $177 \mathrm{Lu}$ dotatate for midgut neuroendocrine tumors. N Engl J Med. 2017 Jan 12;376(2):125-135.

.- The NETTER-1 study demonstrated a favorable safety profile of 177 Lu-DOTATATE.

44. Baum RP, Kluge AW, Kulkarni H, et al. [(177)Lu-DOTA](0)-D-Phe(1)Tyr(3)-Octreotide ((177)Lu-DOTATOC) for peptide receptor radiotherapy in patients with advanced neuroendocrine tumours: a phase-Il study. Theranostics. 2016;6(4):501-510.

45. De Keizer B, Van Aken MO, Feelders RA, et al. Hormonal crises following receptor radionuclide therapy with the radiolabeled somatostatin analogue [177Lu-DOTA0,Tyr3]octreotate. Eur J Nucl Med Mol Imaging. 2008 Apr;35(4):749-755.

46. Cives $M$, Strosberg J. Radionuclide therapy for neuroendocrine tumors. Curr Oncol Rep. 2017 Feb;19(2):9.

47. Imhof A, Brunner P, Marincek N, et al. Response, survival, and longterm toxicity after therapy with the radiolabeled somatostatin analogue [90Y-DOTA]-TOC in metastasized neuroendocrine cancers. J Clin Oncol. 2011 Jun 10;29(17):2416-2423.

48. Valkema R, Pauwels $S$, Kvols LK, et al. Survival and response after peptide receptor radionuclide therapy with [90Y-DOTA0,Tyr3] octreotide in patients with advanced gastroenteropancreatic neuroendocrine tumors. Semin Nucl Med. 2006 Apr;36(2):147-156.

49. Bodei $L$, Kidd M, Paganelli G, et al. Long-term tolerability of PRRT in 807 patients with neuroendocrine tumours: the value and limitations of clinical factors. Eur J Nucl Med Mol Imaging. 2015 Jan;42 (1):5-19.
50. Paganelli G, Sansovini M, Ambrosetti A, et al. 177 Lu-Dota-octreotate radionuclide therapy of advanced gastrointestinal neuroendocrine tumors: results from a phase II study. Eur J Nucl Med Mol Imaging. 2014 Oct;41(10):1845-1851.

51. Bodei $L$, Cremonesi $M$, Ferrari $M$, et al. Long-term evaluation of renal toxicity after peptide receptor radionuclide therapy with $90 \mathrm{Y}$ DOTATOC and 177Lu-DOTATATE: the role of associated risk factors. Eur J Nucl Med Mol Imaging. 2008 Oct;35(10):1847-1856.

52. Garcia-Carbonero R, Rinke A, Valle JW, et al. ENETS consensus guidelines for the standards of care in neuroendocrine neoplasms. Systemic therapy 2: chemotherapy. Neuroendocrinology. 2017 Apr 5.

- The last published ENETS Consensus Guidelines about chemotherapy in neuroendocrine tumors.

53. Strosberg JR, Fine RL, Choi J, et al. First-line chemotherapy with capecitabine and temozolomide in patients with metastatic pancreatic endocrine carcinomas. Cancer. 2011 Jan 15;117(2):268-275.

54. Clewemar Antonodimitrakis P, Sundin A, Wassberg C, et al. Streptozocin and 5-fluorouracil for the treatment of pancreatic neuroendocrine tumors: efficacy, prognostic factors and toxicity. Neuroendocrinology. 2016;103(3-4):345-353.

55. Delaunoit T, Ducreux M, Boige $V$, et al. The doxorubicin-streptozotocin combination for the treatment of advanced well-differentiated pancreatic endocrine carcinoma; a judicious option? Eur J Cancer. 2004 Mar;40(4):515-520.

56. Kouvaraki MA, Ajani JA, Hoff $P$, et al. Fluorouracil, doxorubicin, and streptozocin in the treatment of patients with locally advanced and metastatic pancreatic endocrine carcinomas. J Clin Oncol. 2004 Dec 1;22(23):4762-4771.

57. Turner NC, Strauss SJ, Sarker D, et al. Chemotherapy with 5-fluorouracil, cisplatin and streptozocin for neuroendocrine tumours. $\mathrm{Br} \mathrm{J}$ Cancer. 2010 Mar 30;102(7):1106-1112.

58. Meyer T, Qian W, Caplin ME, et al. Capecitabine and streptozocin \pm cisplatin in advanced gastroenteropancreatic neuroendocrine tumours. Eur J Cancer. 2014 Mar;50(5):902-911.

59. Volkova M, Russell R 3rd. Anthracycline cardiotoxicity: prevalence, pathogenesis and treatment. Curr Cardiol Rev. 2011 Nov;7(4):214220.

- Review of prevalence, pathogenesis and treatment of anthracycline cardiotoxicity.

60. Swain SM, Whaley FS, Ewer MS. Congestive heart failure in patients treated with doxorubicin: a retrospective analysis of three trials. Cancer. 2003 Jun 1;97(11):2869-2879.

61. Ekeblad S, Sundin A, Janson ET, et al. Temozolomide as monotherapy is effective in treatment of advanced malignant neuroendocrine tumors. Clin Cancer Res. 2007 May 15;13(10):2986-2991.

62. Fine RL, Gulati AP, Krantz BA, et al. Capecitabine and temozolomide (CAPTEM) for metastatic, well-differentiated neuroendocrine cancers: the Pancreas Center at Columbia University experience. Cancer Chemother Pharmacol. 2013 Mar;71(3):663-670.

63. Saif MW, Kaley K, Brennan M, et al. A retrospective study of capecitabine/temozolomide (CAPTEM) regimen in the treatment of metastatic pancreatic neuroendocrine tumors (pNETs) after failing previous therapy. Jop. 2013 Sep 10;14(5):498-501.

64. Ramanathan RK, Cnaan A, Hahn RG, et al. Phase II trial of dacarbazine (DTIC) in advanced pancreatic islet cell carcinoma. Study of the Eastern Cooperative Oncology Group-E6282. Ann Oncol. 2001 Aug;12(8):1139-1143.

65. Mueller D, Krug S, Majumder M, et al. Low dose DTIC is effective and safe in pretreated patients with well differentiated neuroendocrine tumors. BMC Cancer. 2016 Aug;18(16):645.

66. Sorbye $\mathrm{H}$, Welin S, Langer SW, et al. Predictive and prognostic factors for treatment and survival in 305 patients with advanced gastrointestinal neuroendocrine carcinoma (WHO G3): the NORDIC NEC study. Ann Oncology. 2013 Jan;24(1):152-160.

- The largest study reporting patient and tumor characteristics, treatment and survival in advanced NEC.

67. Imai $H$, Shirota $H$, Okita A, et al. Efficacy and safety of carboplatin and etoposide combination chemotherapy for extrapulmonary neuroendocrine carcinoma: a retrospective case series. Chemotherapy. 2016;61(3):111-116. 
68. Iwasa S, Morizane C, Okusaka T, et al. Cisplatin and etoposide as first-line chemotherapy for poorly differentiated neuroendocrine carcinoma of the hepatobiliary tract and pancreas. Jpn J Clin Oncol. 2010 Apr;40(4):313-318.

69. Kulke $\mathrm{MH}, \mathrm{Wu} \mathrm{B}$, Ryan DP, et al. A phase II trial of irinotecan and cisplatin in patients with metastatic neuroendocrine tumors. Dig Dis Sci. 2006 Jun;51(6):1033-1038.

70. Lu ZH, Li J, Lu M, et al. Feasibility and efficacy of combined cisplatin plus irinotecan chemotherapy for gastroenteropancreatic neuroendocrine carcinomas. Med Oncol. 2013;30(3):664.

71. Nakano K, Takahashi S, Yuasa T, et al. Feasibility and efficacy of combined cisplatin and irinotecan chemotherapy for poorly differentiated neuroendocrine carcinomas. Jpn J Clin Oncol. 2012 Aug;42(8):697-703.

72. Hadoux J, Malka D, Planchard D, et al. Post-first-line FOLFOX chemotherapy for grade 3 neuroendocrine carcinoma. Endocr Relat Cancer. 2015 Jun;22(3):289-298.

73. Hentic $O$, Hammel $P$, Couvelard A, et al. FOLFIRI regimen: an effective second-line chemotherapy after failure of etoposide-platinum combination in patients with neuroendocrine carcinomas grade 3. Endocr Relat Cancer. 2012 Dec;19(6):751-757.

74. Olsen IH, Sorensen JB, Federspiel B, et al. Temozolomide as second or third line treatment of patients with neuroendocrine carcinomas. ScientificWorldJournal. 2012;2012:170496. 